\title{
Up-regulation of CIT promotes the growth of colon cancer cells
}

\author{
Zehua Wu ${ }^{1, *}$, Xiangying Zhu ${ }^{2,3, *}$, Wendi $\mathbf{X u}^{4}$, Yu Zhang ${ }^{5}$, Lin Chen ${ }^{3}$, Fabo Qiu ${ }^{1}$, \\ Binyuan Zhang ${ }^{1}$, Liqun Wu ${ }^{1}$, Zhihai Peng ${ }^{2}$ and Huamei Tang ${ }^{5}$ \\ ${ }^{1}$ Department of Hepatobiliary Surgery, The Affiliated Hospital of Qingdao University, Qingdao 266003, People's Republic of \\ China \\ ${ }^{2}$ Department of General Surgery, Shanghai Jiaotong University Affiliated First People's Hospital, Shanghai 200080, People's \\ Republic of China \\ ${ }^{3}$ R \& D Department, Shanghai GeneChem Limited Company, Shanghai 201203, People's Republic of China \\ ${ }^{4}$ Department of Continuing Medical Education, The Affiliated Hospital of Qingdao University, Qingdao 266003, People's \\ Republic of China \\ ${ }^{5}$ Department of Pathology, Shanghai Jiaotong University Affiliated First People's Hospital, Shanghai 200080, People's Republic \\ of China \\ *These authors have contributed equally to this work \\ Correspondence to: Huamei Tang, email: huamei-tang@hotmail.com \\ Zhihai Peng, email: zhihai.peng@hotmail.com \\ Keywords: CIT, colon cancer, cell growth, apoptosis, p53
}

Received: July 18, $2016 \quad$ Accepted: May 06, $2017 \quad$ Published: June 27, 2017

Copyright: Wu et al. This is an open-access article distributed under the terms of the Creative Commons Attribution License 3.0 (CC BY 3.0), which permits unrestricted use, distribution, and reproduction in any medium, provided the original author and source are credited.

\section{ABSTRACT}

Colon cancer is one of the major causes of cancer mortality worldwide. However, the underlying mechanism and therapeutic targets of colon cancer have not yet been fully elucidated. In the present study, we demonstrate that citron rho-interacting, serine/threonine kinase 21 (CIT) promotes the growth of human colon cancer cells. CIT is overexpressed in human colon cancer tissues and cell lines. High expression of CIT predicts poor survival for patients with colon cancer. In colon cancer cells, CIT knockdown represses cellular proliferation and colony formation. Our in vivo xenograft experiments showed that CIT knockdown reduces the growth rate of colon cancer cells and the final tumor weight. We found that CIT knockdown induces cell cycle arrest and apoptosis in colon cancer cells. Further microarray and bioinformatics analyses indicated that CIT regulates the p53 signaling pathway, which may account for the effects of CIT on colon cancer cells. Taken together, our findings provide evidence that CIT may promote the development of colon cancer, at least in part, through the p53 signaling pathway. Therefore, CIT may be a potential therapeutic target for colon cancer treatment.

\section{INTRODUCTION}

Colon cancer is one of the major causes of cancer mortality worldwide. Every year, colon cancer affects 1.23 million people and causes 608,000 deaths worldwide $[1,2]$. Globally, the incidence of colon cancer varies considerably and is closely associated with a western lifestyle. Despite strong hereditary components, most cases of colorectal cancer are sporadic and develop slowly over several years through the adenoma-carcinoma sequence [3]. Five-year relative survival is greater than
$90 \%$ for patients with stage I disease. However, for patients with stage IV disease, survival is only slightly greater than $10 \%[3,4]$. The underlying mechanism and therapeutic targets of colon cancer have not yet been fully elucidated.

Citron rho-interacting, serine/threonine kinase 21 (CIT) is present at the cleavage furrow and the midbody during cytokinesis. CIT is essential for cellular abscission $[5,6]$ and can phosphorylate the regulatory light chain of myosin II, which is the primary motor protein responsible for cytokinesis [7, 8]. CIT kinase (CIT-K) controls the 
molecular network required for midbody formation during cytokinesis [9]. CIT-K regulates cytokinesis at a step after Rho in the contractile process [5] by maintaining RhoA localization at the cleavage site, which is necessary for proper RhoA activity and contractile ring dynamics [10]. Nevertheless, the physiological and pathological role of CIT remains largely unknown. In mammals, the loss of CIT-K leads to massive cytokinesis failure and apoptosis in only neuronal progenitors and male germ cells, resulting in severe microcephaly and testicular hypoplasia [11]. However, the cause of this specificity is unknown. Recent studies indicate that CIT plays a key role in the development of human cancer, including hepatocellular carcinoma [12]. However, the role of CIT in human colon cancer remains unknown.

In the present study, we demonstrate that CIT promotes the growth of human colon cancer cells. We first found that CIT was overexpressed in human colon cancer tissues. Then, we knocked down CIT and found that CIT knockdown inhibited the proliferation and colony formation of colon cancer cells in vitro and reduced colon cancer cell growth in vivo. We also found that CIT knockdown regulated the cell cycle and apoptosis partly through the p53 pathway. Therefore, our findings indicate that CIT promotes the growth of human colon cancer cells.

\section{RESULTS}

\section{CIT is overexpressed in human colon cancer tissues and cell lines}

To investigate the potential role of CIT in human colon cancer, we first investigated the expression patterns of CIT in human colon cancer tissues and cell lines. Western blotting revealed that CIT protein levels were significantly up-regulated in 4 tested colon cancer tissues compared with matched adjacent normal tissues (Figure 1A). CIT protein levels were up-regulated in five human colon cancer cell lines compared with the human colon mucosal epithelial cell line NCM460 (Figure 1B). Next, we examined CIT expression in 203 pairs of colon cancer tissues and matched adjacent normal tissues, as well as in 66 lymph node metastasis specimens, using immunohistochemical staining. As shown in Figure 1C, CIT was predominantly localized to the cytoplasm. While CIT staining in normal tissues was $23.2 \%$ positive, CIT staining in cancer tissues was $67.5 \%$ positive, and CIT staining in lymph node metastasis specimens was $69.7 \%$ positive (Figure $1 \mathrm{C}$ and $1 \mathrm{D}, P<0.001$ ), indicating that CIT is up-regulated in colon cancer tissues and metastatic specimens. We next analyzed CIT expression in an independent cohort of colon cancer patients using RNA sequencing in 23 colon tumor tissues and matched adjacent normal tissues. As showed in Figure 1E, the CIT gene was significantly higher expressed in the colon tumor tissues compared with the adjacent normal tissues. Among the 23 enrolled samples, CIT expression was elevated in 22 colon tumor tissues. Taken together, these results indicate that CIT is associated with colon tumorigenesis.

\section{CIT expression is associated with the clinical features of colon cancer}

Next, we investigated the relationship between CIT expression and clinical features of colon cancer patients. We divided the patients into two groups, a CIT high/ positive group $(\mathrm{n}=137)$ and a CIT low/negative group $(n=66)$, based on the immunohistochemical staining results shown in Figure 1D. We found that high CIT levels were strongly associated with high AJCC stage $(P=0.040), \mathrm{T}$ stage $(P=0.008)$ and $\mathrm{M}$ stage $(P=0.011$, Table 1). Moreover, the Kaplan-Meier survival curves and the log-rank test survival analysis showed that the disease-free survival (DFS) and overall survival (OS) of patients with CIT-positive tumors were significantly poorer than those of patients with CIT-negative tumors (DFS: $P=0.002$, Figure 2A; OS: $P<0.001$, Figure 2B). These results suggest that CIT expression is associated with poor survival in patients with colon cancer.

\section{CIT promotes colon cancer cell growth in vitro}

To investigate the role of CIT in human colon cancer, we knocked down CIT with lentivirus-mediated shRNA in RKO and HCT116 cell lines. CIT expression was significantly reduced by sh-CIT in RKO and HCT116 cells at both the mRNA and protein levels (Figure 3A-3D). To study the effects of CIT on cell proliferation, RKO cells were infected with sh-Ctrl or sh-CIT lentivirus, and cell growth was monitored by high-content screening (HCS) every day for five days. As shown in Figure $3 \mathrm{E}$ and $3 \mathrm{~F}$, CIT knockdown significantly suppressed cell growth, and similar results were detected in HCT116 cells using an MTT assay (Figure 3G). Moreover, we analyzed colony formation to determine the contribution of CIT to colon cancer cell tumorigenesis in vitro. The results showed that CIT knockdown in both RKO and HCT116 cells dramatically reduced colony formation (Figure $3 \mathrm{H}$ and 3I). These results show that CIT is necessary for colon cancer cell proliferation and colony formation in vitro.

\section{CIT promotes colon cancer cell growth in vivo}

To investigate the function of CIT in colon cancer cell growth in vivo, we evaluated xenograft formation of HCT116 cells with/without stable CIT knockdown. As expected, after four weeks of injection, the tumors in the CIT knockdown group were significantly smaller than those in the control group (Figure 4A and 4B). Additionally, tumor growth curves were constructed based on the measurements of tumor volumes of the xenografts 
for 28 consecutive days. This analysis revealed that CIT knockdown significantly reduced tumor volume (Figure 4C). Taken together, these data indicate that CIT promotes the xenograft growth of colon cancer cells.

\section{Knockdown of CIT induces cell cycle arrest and apoptosis}

Cell cycle arrest and apoptosis usually have an effect on cell proliferation. To determine whether CIT inhibits colon cancer cell proliferation by regulating the cell cycle and apoptosis, we examined the cell cycle by PI/FACS and detected apoptosis by fluorescence activated cell sorting (FACS). As shown in Figure 5A and $5 \mathrm{~B}$, knockdown of CIT expression led to cell cycle arrest in the G0/G1 phase (47.3\% for sh-Ctrl vs. $57.7 \%$ for sh-CIT). Additionally, CIT knockdown induced cell cycle arrest in HCT116 cells (Supplementary Figure 1). Furthermore, the percentage of apoptotic RKO cells was significantly increased in the CIT knockdown group compared with the control group (Figure 5C and 5D, $4.35 \%$ for sh-Ctrl vs. $15.46 \%$ for sh-CIT), and similar results were observed in HCT116 cells (Figure 5E and $5 \mathrm{~F}$ ). These results indicate that CIT regulates the cell cycle and apoptosis of colon cancer cells.

\section{Knockdown of CIT suppresses the p53 and apoptosis pathways}

The above results showed that CIT was required for colon cancer cell growth in vitro and in vivo, partly by regulating colon cancer cell cycle and survival. However,
A

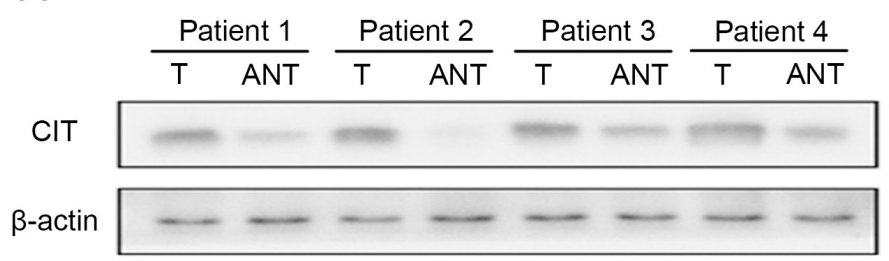

C
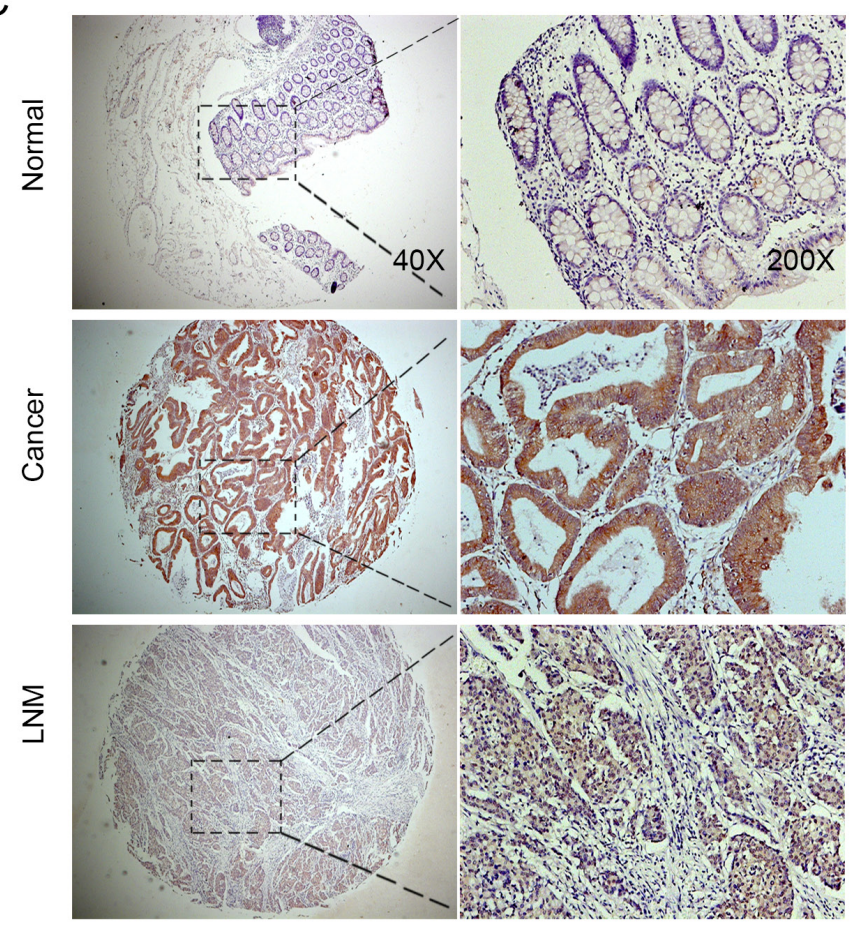

B

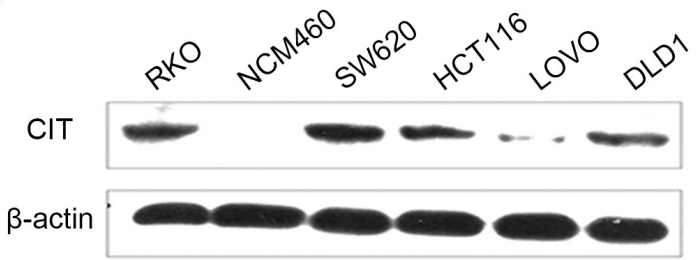

D

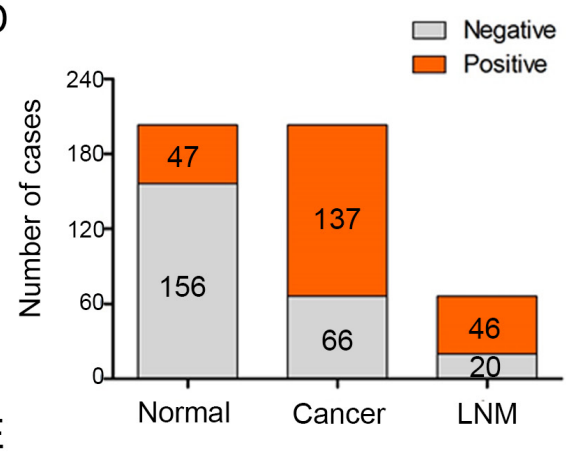

$E$

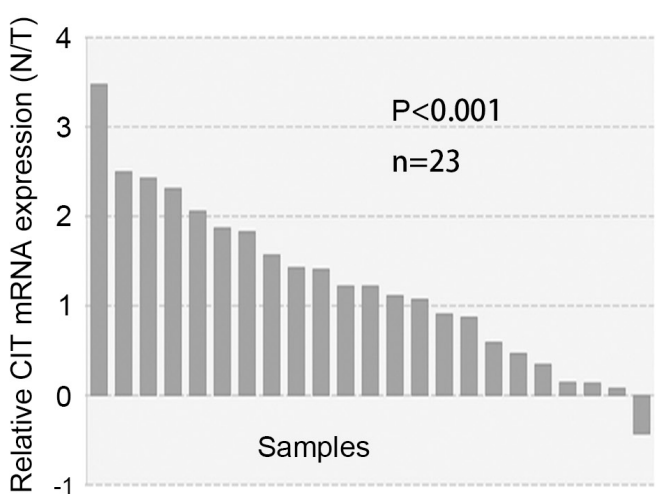

Figure 1: CIT is overexpressed in human colon cancer. (A) Western blot analysis of CIT in primary colon cancer tissues (T) and matched adjacent noncancerous tissues (ANT). $\beta$-Actin was used as a loading control. (B) Western blot analysis of CIT in the human colon mucosal epithelial cell line NCM460 and in five colon cancer cell lines. $\beta$-Actin was used as a loading control. (C) Immunohistochemical (IHC) staining analysis of CIT protein expression in primary colon cancer tissues, matched adjacent noncancerous tissues, and lymph node metastasis specimens. (D) A schematic representation of CIT expression from colon cancer tissue microarrays evaluated by IHC staining in (C). (E) Relative CIT expression levels in colon cancer tissues and the matched adjacent tissues of the patients included in the Cancer Genome Atlas (TCGA) database ( $\mathrm{n}=23)$. 
Table 1: CIT expression and clinicopathologic characteristics

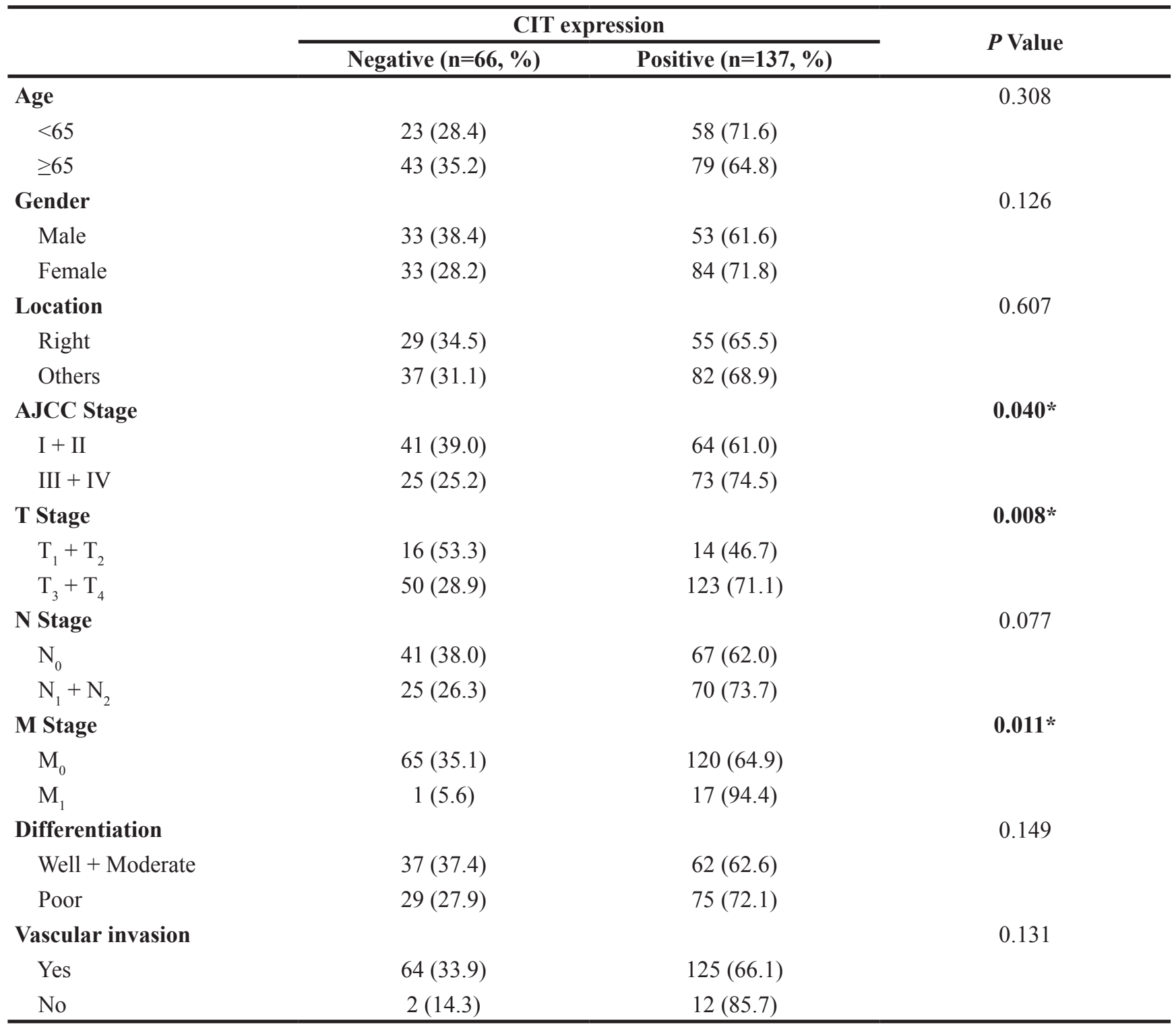

A

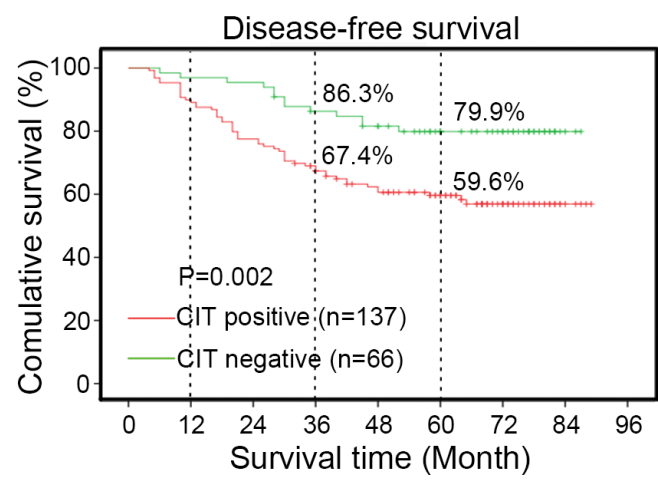

B

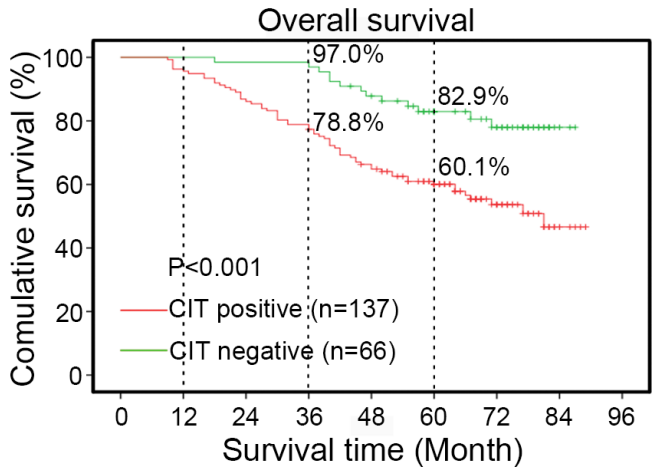

Figure 2: Kaplan-Meier curves based on CIT expression. (A-B) Disease-free survival (DFS) and overall survival (OS) curves of 203 primary colon tumors. The survival rate of the CIT-positive group $(\mathrm{n}=137)$ is significantly lower than that of the CIT-negative group $(\mathrm{n}=66)$. DFS $\mathrm{P}=0.002$; OS $\mathrm{P}<0.001$. 
A

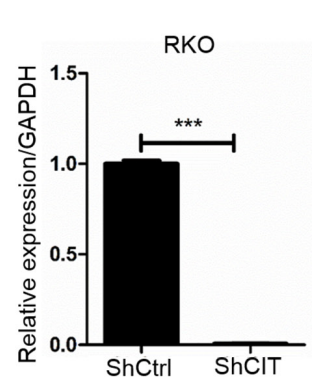

E
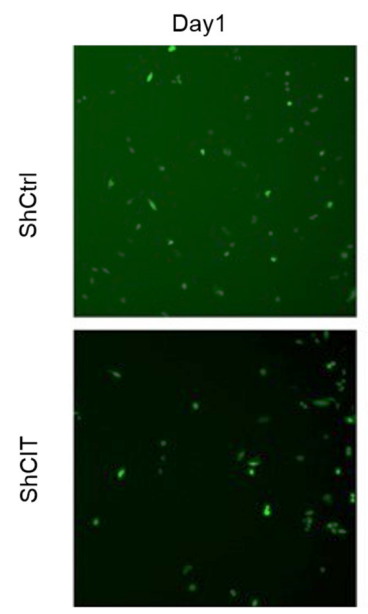

F

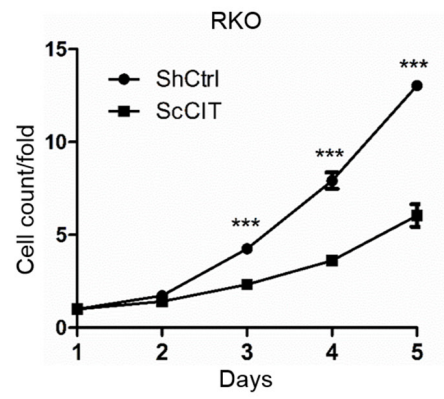

G

HTC116

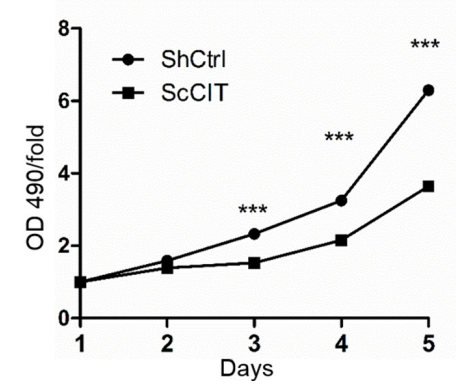

C

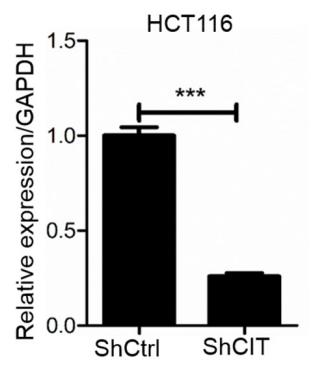

D

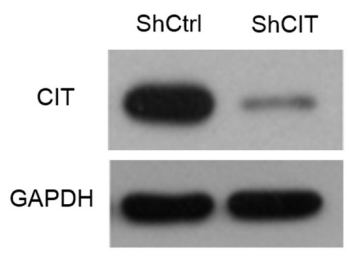

HCT116

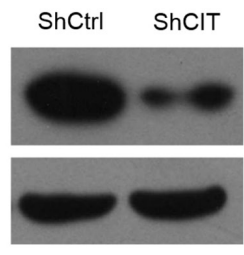

Day2
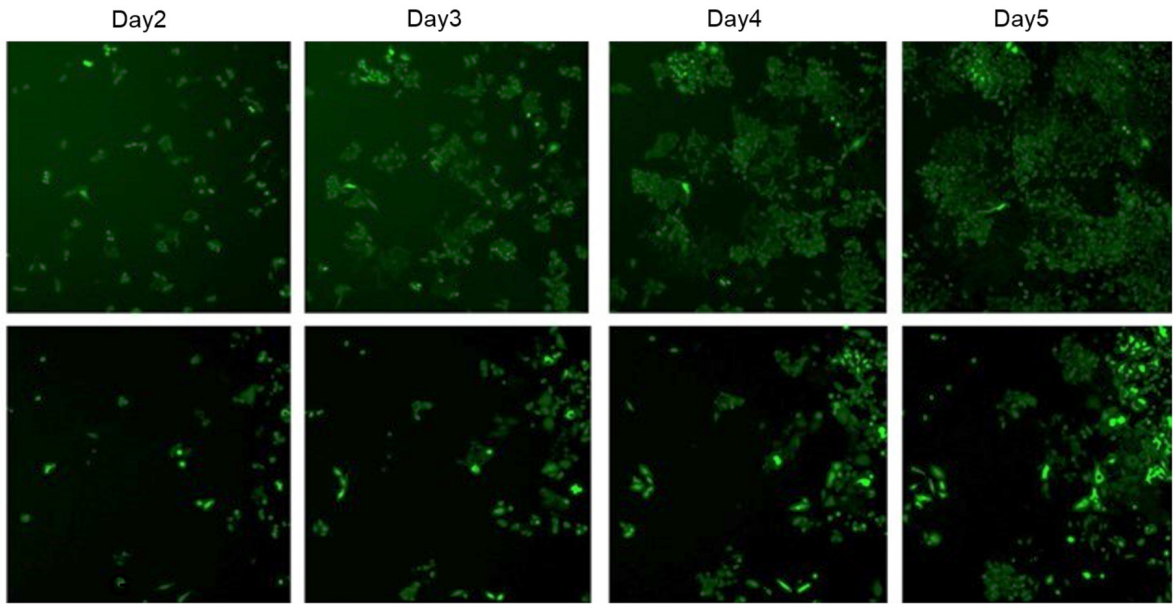

H
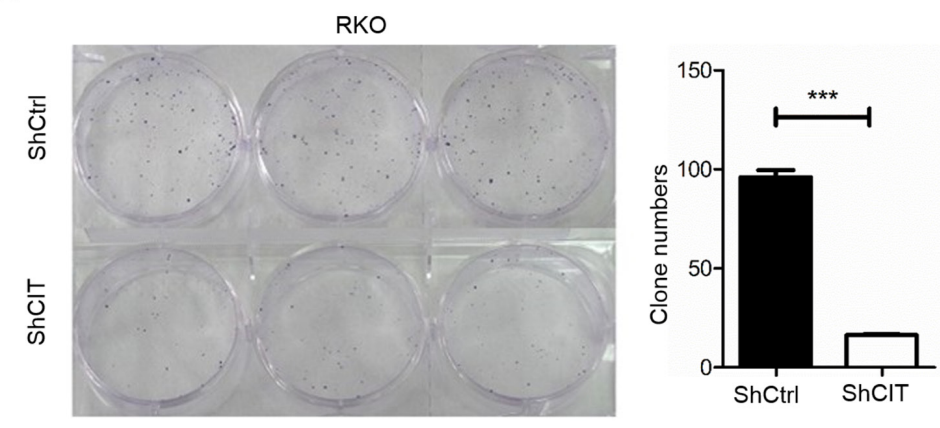

I

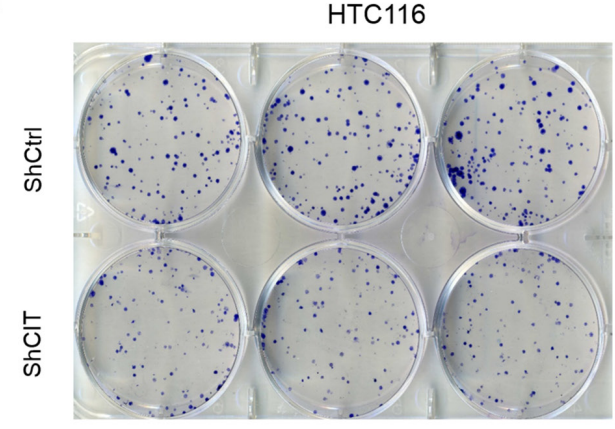

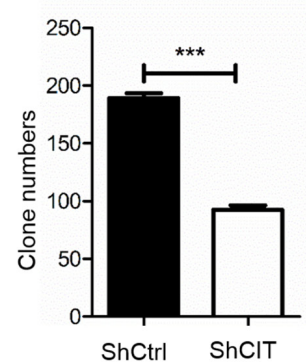

Figure 3: Knockdown of CIT inhibits human colon cancer cell growth in vitro. (A-B) Quantitative RT-PCR and western blot analysis show that CIT expression was efficiently knocked down in the RKO cells. (C-D) Quantitative RT-PCR and western blot analysis show that CIT expression was efficiently knocked down in the HCT116 cells. (E) Representative pictures of RKO cells infected with Control-shRNA (top) and CIT-shRNA (bottom) via multiparametric high-content screening (HCS) every day for five days. (F) CIT knockdown inhibits the proliferation of RKO cells. $* * * \mathrm{P}<0.001$ vs. sh-Ctrl. (G) CIT knockdown inhibits the proliferation of HCT116 cells. ***P $<0.001$ vs. sh-Ctrl. (H-I) Colony formation assay of RKO and HCT116 cells after infection with sh-Ctrl and sh-CIT lentivirus. $* * * \mathrm{P}<0.001$ vs. sh-Ctrl. 
A
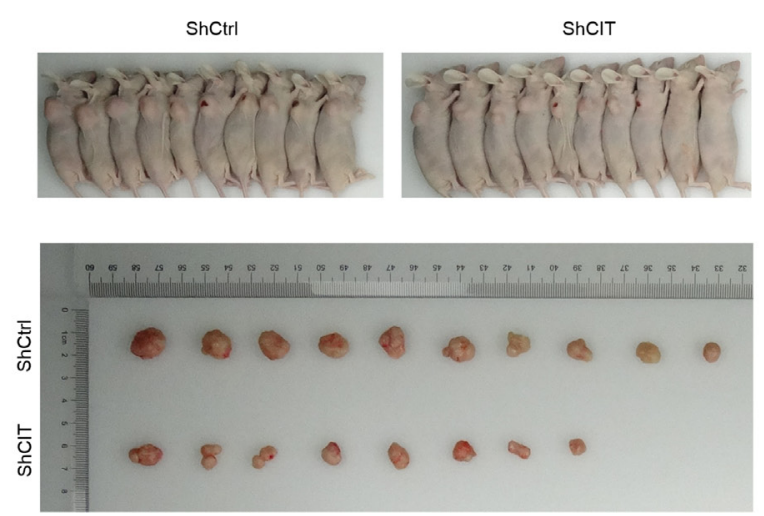
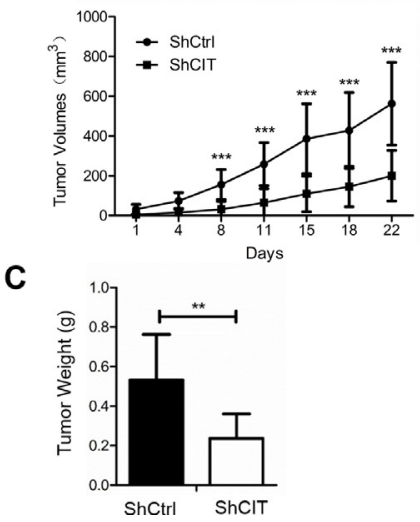

Figure 4: CIT knockdown represses colon cancer cell growth in vivo. (A) Subcutaneous tumors in nude mice and isolated tumors after 4 weeks formed by injection of RKO cells infected with sh-Ctrl and sh-CIT lentivirus ( $\mathrm{n}=10$ in each group). (B) Tumor growth curves of xenografts in nude mice ( $\mathrm{n}=10$ in each group). Xenograft volumes were calculated using the formula $\mathrm{v}=0.5 \mathrm{ab}^{2}$ (a: long diameter, $\mathrm{b}$ : short diameter). $* * * \mathrm{P}<0.001$ vs. sh-Ctrl. (C) Statistics for the tumor weights of the xenografts ( $\mathrm{n}=10$ in each group). $* * \mathrm{P}<0.01$ vs. Sh-Ctrl.

A

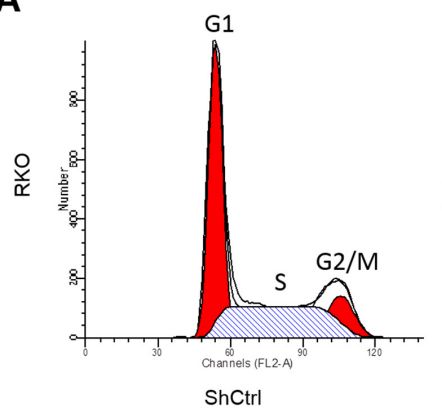

C

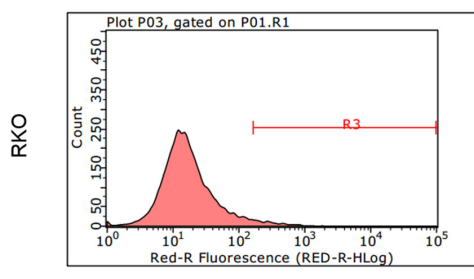

ShCtrl

E

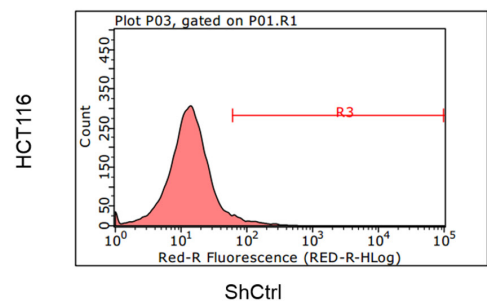

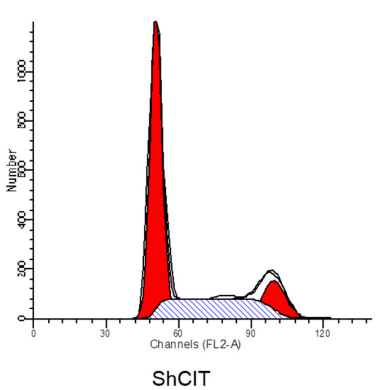

$\mathrm{ShClT}$

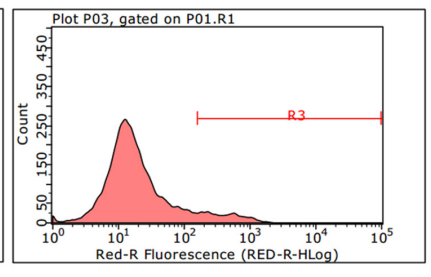

$\mathrm{ShClT}$

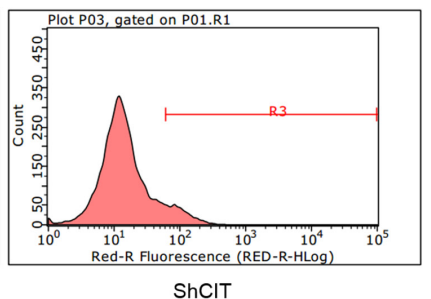

B

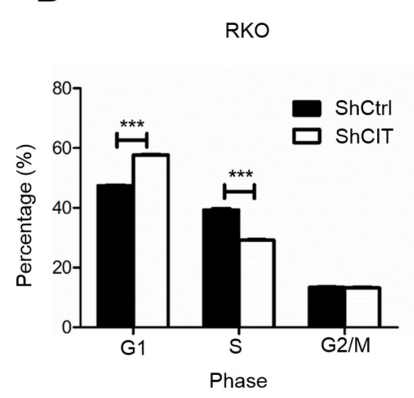

D

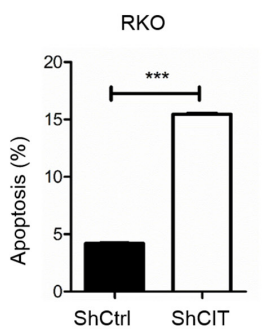

$\mathbf{F}$

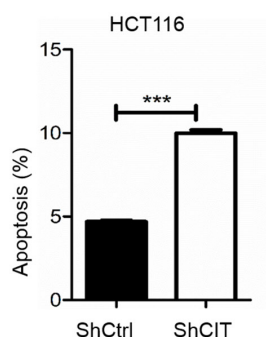

Figure 5: Knockdown of CIT induces cell cycle arrest and apoptosis. (A-B) Flow cytometry analysis of the cell cycle reveals that CIT knockdown induces RKO cell cycle arrest at the G1 phase (G1 phase: $47.34 \% \pm 0.52 \%$ vs. $57.72 \% \pm 0.40 \%$; S phase: $39.28 \%$ $\pm 0.78 \%$ vs. $29.14 \% \pm 0.34 \%$; and G2/M phase: $13.38 \% \pm 0.31 \%$ vs. $13.14 \% \pm 0.53 \%$ ). ${ }^{*} * * \mathrm{P}<0.001$ vs. sh-Ctrl. (C-D) Flow cytometry analysis of apoptosis reveals that the rates of apoptosis of RKO cells are significantly increased by CIT knockdown $(5.02 \% \pm 0.29 \%$ vs. $19.60 \% \pm 0.29 \%$ ). ***P $<0.001$ vs. sh-Ctrl. (E-F) Flow cytometry analysis of apoptosis reveals that the rates of apoptosis of HCT116 cells are significantly increased by CIT knockdown $(4.70 \% \pm 0.13$ vs. $9.99 \% \pm 0.34 \%)$. ${ }^{* * *} \mathrm{P}<0.001$ vs. sh-Ctrl. 
the mechanisms underlying CIT-mediated phenotypes in colon cancer cells were still unknown. To address this, global gene expression profiling was analyzed in RKO cells with/without CIT knockdown (Figure 5A). We found that 295 genes were up-regulated and that 590 genes were down-regulated by CIT knockdown (Figure 6A). Next, we performed a KEGG pathway analysis and found that the differentially expressed genes were enriched in ten pathways (Figure 6B). The p53 signaling pathway was the most significantly enriched pathway in cells with CIT knockdown ( $P=1.44 \mathrm{E}-06$, Figure 6B). Next, we analyzed the functional interaction network between CIT and the p53 signaling pathway. As shown in Figure 6C, cell cycle regulators (CCND1, CCNG1, CCNG2, CDK1, SESN2 and RRM2) of the p53 signaling pathway were downregulated by CIT knockdown.

To further understand the intracellular signaling pathway induced by CIT in colon cancer cells, we analyzed key proteins involved in cell proliferation, cell growth, cell cycle regulation and apoptosis using the PathScan ${ }^{\circledR}$ intracellular signaling array kit. Interestingly, CIT knockdown could significantly promote the phosphorylation of Bad and the cleavage of caspase-3 (Figure 6D). Finally, we validated by western blotting
A

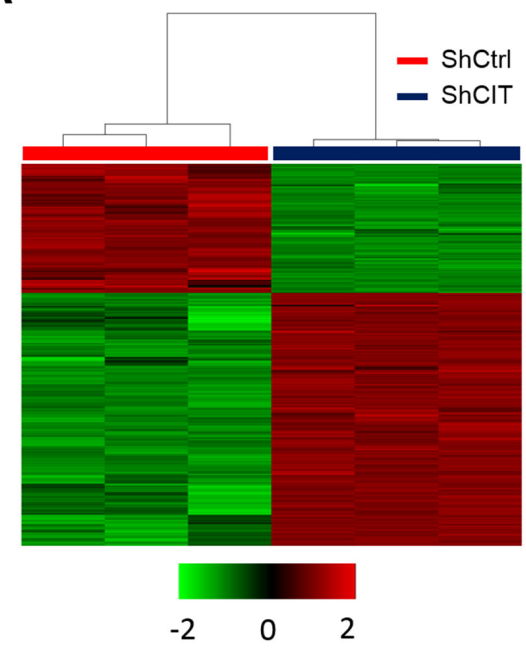

B

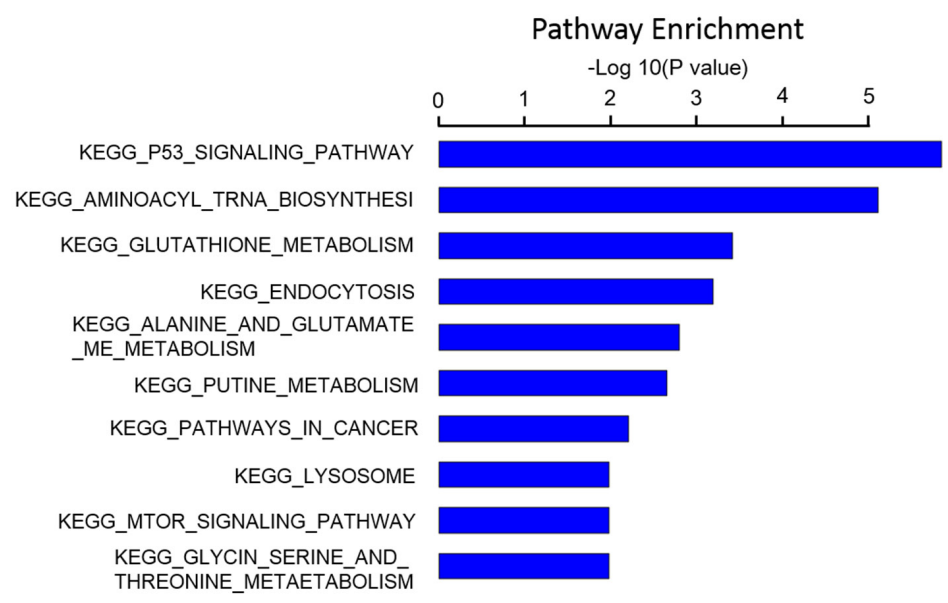

$\mathbf{E}$

C

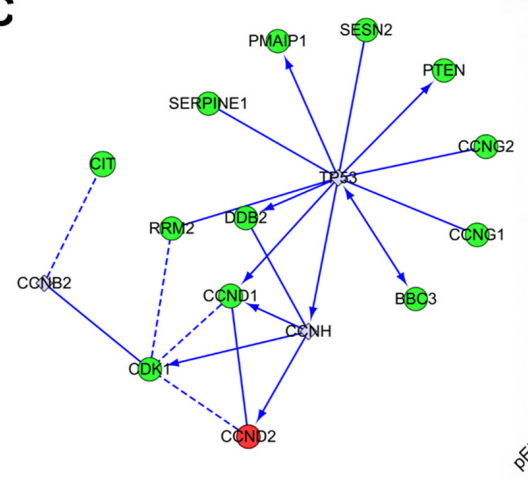

D

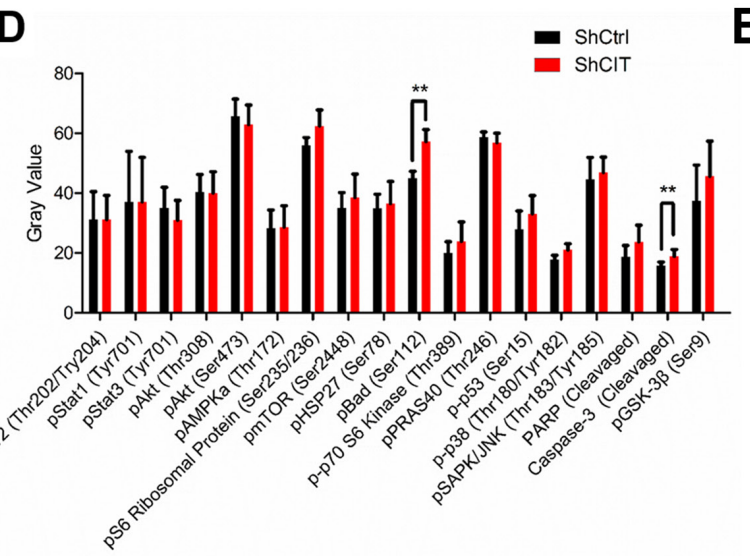

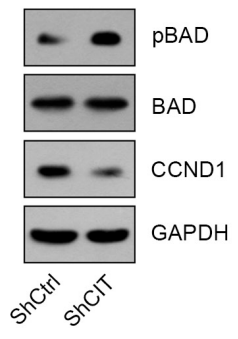

Figure 6: Down-regulation of CIT affects the p53 and apoptosis pathways. (A) Heat map of 885 genes (295 genes up-regulated and 590 genes down-regulated) showing significant differential expression patterns in RKO cells infected with sh-Ctrl or sh-CIT lentivirus (criteria: $P<0.05$ and absolute fold change $>1.5$ ). (B) Functional pathway enrichment of differential genes analyzed based on the KEGG and BIOCARTA databases. The P53 signaling pathway was the most significantly enriched pathway $(P=1.44 \mathrm{E}-06)$. (C) Interaction network between CIT and the genes involved in the KEGG pathway P53. Green circles represent down-regulated genes, red circles represent upregulated genes, and gray circles represent genes with no change in expression. (D) The post-translational modification of 18 important signaling proteins (16 phosphorylation proteins and 2 cleavage proteins) was monitored by array hybridization. CIT knockdown increases Bad phosphorylation and Caspase- 3 cleavage in RKO cells. $* * P<0.01$ vs. sh-Ctrl. (E) CIT knockdown regulates Bad and CCND1. RKO cells were infected with sh-CIT or sh-Ctrl for $48 \mathrm{~h}$. Then, cell lysates were subjected to western blotting with the indicated antibodies. GAPDH was used as an internal control. 
that CIT knockdown up-regulated p-Bad and downregulated CCND1 in RKO cells (Figure 6E). These results indicate that CIT knockdown suppressed colon cancer tumorigenesis via the p53 and apoptosis pathways.

\section{DISCUSSION}

In the present study, we demonstrate that CIT acts as a tumor promoter in human colon cancer. CIT expression is up-regulated in human colon cancer tissues, and upregulation of CIT is associated with advanced disease stage and poor survival. Using loss-of-function experiments, we show that CIT knockdown inhibits proliferation and colony formation of colon cancer cells in vitro and growth of colon cancer cells in vivo. Our investigation of the mechanism indicates that CIT knockdown induces cell cycle arrest and apoptosis in colon cancer cells. The microarray data and bioinformatics analysis show that CIT regulates the p53 pathway, which may account for the effects of CIT on colon cancer cells.

$\mathrm{Fu}$ et al. [12] reported that CIT is frequently upregulated in human hepatocellular carcinoma compared with adjacent non-tumor tissues. Another study showed that the down-regulation of miR-486 mediates the upregulation of CIT in hepatocellular carcinoma [15]. In this study, we show that CIT expression is markedly up-regulated in human colon cancer, indicating that CIT may facilitate the development of colon cancer. Yan et al. [16] identified and analyzed the epigenetically silenced microRNAs in colorectal cancer cells. They found that miR-1247, which is predicted to target CIT (http://www. microrna.org), was significantly silenced in colon cancer cells [16]. Therefore, the lack of miR-1247 may result in increased CIT expression in colon cancer tissues. However, further experimental evidence is needed to confirm this.

We found that CIT knockdown inhibits proliferation and colony formation in the colon cancer cell lines RKO and HCT116. Additionally, our xenograft experiments revealed that CIT knockdown repressed colon cancer cell growth in vivo. CIT is present at the cleavage furrow and midbody during cytokinesis and is essential for cellular abscission. CIT also regulates the G2/M transition in rat hepatocytes, and knocking down CIT inhibits the proliferation of hepatocellular carcinoma cells [12]. We found that CIT knockdown also induced cell cycle arrest in colon cancer cells. However, while CIT knockdown inhibits the G1/S transition in colon cancer cells, CIT knockdown does not inhibit the G2/M transition as is observed in hepatocellular carcinoma cells. Furthermore, the percentage of apoptotic cells was significantly upregulated when CIT was knocked down in colon cancer cells. These findings indicate that CIT regulates the cell cycle and cell survival to promote the development of colon cancer.
The tumor suppressor p53 is a transcription factor that regulates several genes with a broad range of functions, including DNA repair, metabolism, cell cycle arrest, apoptosis and senescence [17, 18]. Many different types of cancer are associated with a high incidence of TP53 mutations, leading to the expression of mutant p53 proteins [19]. There is growing evidence that these mutant p53 proteins have lost wild-type p53 tumor suppressor activity $[20,21]$. Alterations in the $\mathrm{p} 53$ pathway play a significant role in colon cancer [22]. Interestingly, our microarray and bioinformatics analyses show that CIT knockdown in RKO cells activates genes in the p53 pathway, indicating that p53 signaling plays a pivotal role in the effects of CIT on the cell cycle and apoptosis, which subsequently regulate cell growth and cancer development, of colon cancer cells.

In summary, we provide evidence that CIT is overexpressed in human colon cancer tissues and serves as a tumor promoter. CIT maintains the cell cycle and survival partly by regulating the p53 signaling pathway. Therefore, CIT may be a potential therapeutic target for treating human colon cancer.

\section{MATERIALS AND METHODS}

\section{Patient information}

All 203 patients with colon cancer, including 86 men and 117 women, were enrolled from 2001 to 2003. The median age of the patients was 68 years (range: 2295 ) at the time of surgery, and the median follow-up time was 61 months post-operatively (range: 9-89 months). The adjacent normal tissues were taken from an area more than $10 \mathrm{~cm}$ away from the primary neoplasms, and paired lymph node metastasized specimens were taken from 66 patients. The study was approved by the Ethics Committee of Shanghai Jiaotong University affiliated with the First People's Hospital. Written informed consent was obtained from all patients.

\section{Colon cancer mRNA database and analysis}

Transcriptome expression datasets and the corresponding clinical information were downloaded from The Cancer Genome Atlas (http://cancergenome.nih. gov). A total of 23 samples, which included transcriptional expression data for both tumor tissues and adjacent normal tissues, were available for this analysis.

\section{Cell lines and cell culture}

The human colon mucosa cell line NCM460 was obtained from INCELL (San Antonio, USA). The human colon cancer cell lines RKO, DLD-1, SW620, LoVo and HCT116 were purchased from the Type Culture Collection of the Chinese Academy of Science (Shanghai, 
China). Cells were cultured in Dulbecco's modified Eagle's Medium (DMEM)/F12 (Gibco, Invitrogen Corp., San Diego, California, USA) combined with $10 \%$ fetal bovine serum and $1 \%$ penicillin/streptomycin at $37^{\circ} \mathrm{C}$ in a humidified atmosphere of $5 \% \mathrm{CO}_{2}$ and $95 \%$ air.

\section{RNA Isolation and quantitative real-time PCR}

Total RNA was extracted and purified using Trizol reagent (Invitrogen) according to the manufacturer's instructions. Reverse transcription was performed using M-MLV reverse transcriptase (Promega) and random primers (Sangon, Shanghai, China) to obtain cDNAs. CIT mRNA expression was analyzed by quantitative real-time PCR using SYBR master mix (Takara) on a real-time PCR machine TP800 (Takara). The sequences of the primers used are as follows: CIT forward, 5'-CAGGCAAGATTGAGAACG-3'; CIT reverse, 5'-GCACGATTGAGACAGGGA-3'; GAPDH forward, 5'-TGACTTCAACAGCGACACCCA-3'; and GAPDH reverse, 5'-CACCCTGTTGCTGTAGCCAAA-3. The relative CIT expression was normalized to GAPDH, and data analysis was conducted using the comparative CT method.

\section{Western blot}

Cells were washed with PBS and lysed with lysis buffer (Beyotime). Total protein levels were measured using a BCA protein assay kit (Pierce). Western blotting was performed as described previously [13]. Briefly, 20 $\mu \mathrm{g}$ of total protein was mixed with $2 \times$ loading buffer and separated by $10 \%$ sodium dodecyl sulfate polyacrylamide gel electrophoresis (SDS-PAGE). The proteins were then transferred to polyvinylidene fluoride (PVDF) membranes (Amersham). The PVDF membranes were blocked with $5 \%$ fat-free milk dissolved in Tris-buffered saline and Tween 20 (TBST) buffer for $1 \mathrm{~h}$ and then incubated with the primary antibodies (anti-CIT, Abcam, ab110897; antiCCND1, Abcam, ab16663; anti-pBad, Cell Signaling Technology (CST), \#9664; anti-Bad, Abcam, ab32445; and anti-GAPDH, Santa Cruz Biotechnology, sc-32233) overnight at $4^{\circ} \mathrm{C}$. After three washes with TBST buffer, the secondary antibody (Santa Cruz Biotechnology, sc-2005) was added, and immune activity was detected using an ECL-Plus kit (Amersham Biosciences).

\section{Tissue microarray construction and immunohistochemistry}

Tissue microarrays of 203 pairs of colon cancer and adjacent tissues, as well as 66 lymph node metastases, were performed using immunohistochemical staining. CIT immunoreactivity was assessed independently by two expert pathologists blinded to the clinical data, and the scoring standard used has been described previously [14].
The staining intensity and extent of CIT expression were graded as follows: negative $=$ score 0 , weak $=$ score 1 , moderate $=$ score 2 , and strong $=$ score 3 . The extent of staining was categorized by the percentage of strongly stained cells in the cancer nest as follows: negative $=$ score 0,1 to $25 \%=$ score 1,26 to $50 \%=$ score 2,51 to $75 \%=$ score 3 and 76 to $100 \%=$ score 4 . CIT scoring was assigned to each tumor by multiplying the staining intensity score and the staining extent score. Negative staining refers to a CIT score $\leq 6$, and positive staining refers to a CIT score $>6$.

\section{Packaging of sh-CIT lentivirus}

The lentivirus vector system included the following vectors: pGCSIL-GFP, which stably expresses a short hairpin RNA (shRNA) and a marker (GFP fusion protein); pHelper1.0 (gag/pol element); and Helper2.0 (VSVG element). The shRNA targeting human CIT (5'-GCGTCCTCATACCAGGATAAA-3') and the negative control (NC) shRNA (5' - TTCTCCGAACGTGTCACGT-3') were designed, synthesized and cloned into the pGCSIL-GFP vector by the GeneChem Corporation (Shanghai, China). The pGCSIL-shRNA-GFP, pHelper1.0 and Helper2.0 were mixed and transfected into 293T cells using Lipofectamine TM 2000 (Invitrogen, Shanghai, China) according to the manufacturer's instructions. After transfection for $48 \mathrm{~h}$, the viral supernatants were collected, centrifuged, and then filtered through a $0.45 \mu \mathrm{m}$ polyvinylidene fluoride membrane.

\section{High-content screening cell proliferation assay}

Cell growth was measured via multiparametric highcontent screening (HCS). After $72 \mathrm{~h}$ of infection with the NC or CIT-shRNA lentivirus, RKO cells were harvested, re-suspended, counted and inoculated into 96-well plates at $37^{\circ} \mathrm{C}$ with $5 \% \mathrm{CO}_{2}$ for 5 days. The proliferation of the cells in each of the wells was analyzed daily using ArrayScan ${ }^{\mathrm{TM}}$ HCS software (Cellomics Inc.). The system uses a computerized, automated fluorescence imaging microscope that automatically identifies stained cells and measures the intensity and distribution of fluorescence in each individual cell. Images were acquired for each fluorescence channel using suitable filters and a $20 \times$ objective. Images and data were stored in a Microsoft SQL database.

\section{MTT cell proliferation assay}

Cells infected with the NC or CIT-shRNA lentivirus were seeded in 96-well plates at a density of $2 \times 10^{3}$ cells/ well and incubated at $37^{\circ} \mathrm{C}$ for $1,2,3,4$, or 5 days. Then, the cells were washed two times with PBS, and 3-(4,5-dimethyl2-yl)-2,5-diphenyltetrazolium bromide (MTT) solution (5 $\mathrm{mg} / \mathrm{mL}$ ) was added to each well. After $4 \mathrm{~h}$ of incubation, the supernatants were removed, and then $100 \mu \mathrm{L}$ of dimethyl sulfoxide (DMSO) was added to solubilize the formazan 
salt. Ten minutes later, the optical density (OD) was measured at $490 \mathrm{~nm}$ using a microplate reader.

\section{Colony formation assay}

Cells infected with the NC or CIT-shRNA lentivirus were seeded in six-well plates ( 800 cells/well). The media was replaced every 2 to 3 days. After incubation at $37^{\circ} \mathrm{C}$ for 14 days, adherent cells were washed twice with PBS and then fixed with $4 \%$ paraformaldehyde for 30 minutes. The colonies were stained with Giemsa solution for $10 \mathrm{~min}$ and then washed twice with double distilled water. The number of colonies (> 50 cells/colony) were counted using a fluorescence microscope (CKX41, Olympus, Tokyo, Japan).

\section{Apoptosis assay}

Apoptosis was analyzed by staining with Annexin V-APC (eBioscience) according to the manufacturer's protocol. Cells were infected with the NC or CIT-shRNA lentivirus. After incubation for another 4 days, the cells were harvested, washed with PBS, and resuspended using staining buffer at a final density of $1 \times 10^{6}-1 \times 10^{7} / \mathrm{ml}$. Next, $5 \mu \mathrm{l}$ annexin $\mathrm{V}$-APC was added to $100 \mu \mathrm{l}$ cell suspensions Then, the cell suspensions were incubated at room temperature for $15 \mathrm{~min}$ and subjected to flow cytometry (FACSCalibur, Becton-Dickinson, USA).

\section{Cell cycle assay}

Cells were infected with the NC or CIT-shRNA lentivirus. After incubation for $96 \mathrm{~h}$, cells were seeded in six-well culture plates and cultured to $80 \%$ confluence. Cells were fixed with $70 \%$ cold ethanol at $4^{\circ} \mathrm{C}$ overnight, washed twice with ice-cold PBS, and incubated with 10 $\mathrm{mg} / \mathrm{ml} \mathrm{RNase}$ at $37^{\circ} \mathrm{C}$. The cell cycle was monitored by propidium iodide (PI) staining of the nucleus. The fluorescence of DNA-bound PI in cells was measured by flow cytometry (FACSCalibur, Becton Dickinson). The dot plots and gating strategy of FACS analyses were showed in Supplementary Figure 2.

\section{In vivo xenograft assay}

Four-week-old male nude mice were used for the xenograft growth curve assay. A total of $4 \times 10^{6}$ HCT116 cells were subcutaneously injected into the right armpits of the mice, and the xenograft diameters were measured using a slide caliper rule every other day for 28 days. The xenograft tumor volume was calculated using the following formula: $\mathrm{v}=0.5 \mathrm{ab}^{2}$ (where $\mathrm{a}=$ long diameter of the tumor, $b=$ short diameter of the tumor, and $v=$ volume).

\section{Microarray}

Total RNA from RKO cells infected with the $\mathrm{NC}(\mathrm{n}=3)$ or sh-CIT lentivirus ( $\mathrm{n}=3$ ) was extracted using Trizol reagent.
A NanoDrop 2000 and an Agilent Bioanalyzer 2100 were used to detect the RNA quantity and quality, respectively. Microarrays were processed to generate gene expression profiles using the Affymetrix human GeneChip PrimeView assay according to the manufacturer's instructions. Genes that were differentially expressed between RKO cells treated with sh-Ctrl and those treated with sh-CIT were identified based on the following criteria: $\mathrm{P}<0.05$ and an absolute fold change $>1.5$. Pathway enrichment analyses using the KEGG and BIOCARTA databases were performed for all significantly differentially expressed genes.

\section{Intracellular signaling assay}

To determine the intracellular signaling pathway that is responsible for the phenotype induced by CIT, we detected modifications in a set of cellular proteins that play known roles in cell proliferation and apoptosis using a PathScan ${ }^{\circledR}$ intracellular signaling array kit (CST, \#7323). RKO cell lysates were prepared and analyzed according to the protocol provided by CST. Each experiment was repeated three times.

\section{Statistical analysis}

The statistical analyses were performed using SPSS version 16.0 (SPSS, Inc., Chicago, IL). The raw data are presented as the mean \pm standard error of mean (SEM) of at least three independent experiments, unless otherwise stated. Student's $t$ tests were applied to analyze the differences between two groups. Differences among groups were determined by one-way or two-way analysis of variance (ANOVA) with repeated measures, followed by the Bonferroni post hoc test. A P value of less than 0.05 was considered significant.

\section{Author contributions}

Zehua Wu, Xiangying Zhu, Wendi Xu, Yu Zhang, Lin Chen, Fabo Qiu, Binyuan Zhang, Liqun Wu, Zhihai Peng \& Huamei Tang designed the study. All authors performed the research and interpreted the results. Zehua Wu, Xiangying Zhu, Wendi Xu \& Lin Chen wrote the manuscript. All authors approved the final manuscript.

\section{ACKNOWLEDGMENTS}

This project was supported by funds from the National Natural Science Foundation of China (81472238 \& 81301950) and Important International Cooperation grants from the National Natural Science Foundation of China (81220108021).

\section{CONFLICTS OF INTEREST}

There are no conflicts of interest to disclose. 


\section{REFERENCES}

1. Ku G, Tan IB, Yau T, Boku N, Laohavinij S, Cheng AL, Kang YK, de Lima Lopes G Jr. Management of colon cancer: resource-stratified guidelines from the Asian Oncology Summit 2012. The Lancet Oncol. 2012; 13:e470-481.

2. Okugawa Y, Grady WM, Goel A. Epigenetic alterations in colorectal cancer: emerging biomarkers. Gastroenterology. 2015; 149:1204-1225.e12.

3. Brenner H, Kloor M, Pox CP. Colorectal cancer. Lancet. 2014; 383:1490-1502.

4. Dienstmann R, Salazar R, Tabernero J. Personalizing colon cancer adjuvant therapy: selecting optimal treatments for individual patients. J Clin Oncol. 2015; 33:1787-1796.

5. Madaule P, Eda M, Watanabe N, Fujisawa K, Matsuoka T, Bito H, Ishizaki T, Narumiya S. Role of citron kinase as a target of the small GTPase Rho in cytokinesis. Nature. 1998; 394:491-494.

6. Paramasivam M, Chang YJ, LoTurco JJ. ASPM and citron kinase co-localize to the midbody ring during cytokinesis. Cell Cycle. 2007; 6:1605-1612.

7. Yamashiro S, Totsukawa G, Yamakita Y, Sasaki Y, Madaule P, Ishizaki T, Narumiya S, Matsumura F. Citron kinase, a Rho-dependent kinase, induces di-phosphorylation of regulatory light chain of myosin II. Mol Biol Cell. 2003; 14:1745-1756.

8. De Lozanne A, Spudich JA. Disruption of the Dictyostelium myosin heavy chain gene by homologous recombination. Science. 1987; 236:1086-1091.

9. Bassi ZI, Audusseau M, Riparbelli MG, Callaini G, D'Avino PP. Citron kinase controls a molecular network required for midbody formation in cytokinesis. Proc Natl Acad Sci U S A. $2013 ; 110: 9782-9787$.

10. Bassi ZI, Verbrugghe KJ, Capalbo L, Gregory S, Montembault E, Glover DM, D'Avino PP. Sticky/citron kinase maintains proper RhoA localization at the cleavage site during cytokinesis. J Cell Biol. 2011; 195:595-603.

11. Di Cunto F, Imarisio S, Hirsch E, Broccoli V, Bulfone A, Migheli A, Atzori C, Turco E, Triolo R, Dotto GP, Silengo L, Altruda F. Defective neurogenesis in citron kinase knockout mice by altered cytokinesis and massive apoptosis. Neuron. 2000; 28:115-127.
12. Fu Y, Huang J, Wang KS, Zhang X, Han ZG. RNA interference targeting CITRON can significantly inhibit the proliferation of hepatocellular carcinoma cells. Mol Biol Rep. 2011; 38:693-702.

13. An XZ, Zhao ZG, Luo YX, Zhang R, Tang XQ, Hao DL, Zhao X, Lv X, Liu DP. Netrin-1 suppresses the MEK/ERK pathway and ITGB4 in pancreatic cancer. Oncotarget. 2016; 7:24719-24733. doi: 10.18632/oncotarget.8348.

14. Li D, Wei P, Peng Z, Huang C, Tang H, Jia Z, Cui J, Le X, Huang S, Xie K. The critical role of dysregulated FOXM1PLAUR signaling in human colon cancer progression and metastasis. Clin Cancer Res. 2013; 19:62-72.

15. Sun H, Cui C, Xiao F, Wang H, Xu J, Shi X, Yang Y, Zhang Q, Zheng X, Yang X, Wu C, Wang L. miR-486 regulates metastasis and chemosensitivity in hepatocellular carcinoma by targeting CLDN10 and CITRON. Hepatol Res. 2015; 45:1312-1322.

16. Yan H, Choi AJ, Lee BH, Ting AH. Identification and functional analysis of epigenetically silenced microRNAs in colorectal cancer cells. PLoS One. 2011; 6:e20628.

17. Khoo KH, Verma CS, Lane DP. Drugging the p53 pathway: understanding the route to clinical efficacy. Nat Rev Drug Discov. 2014; 13:217-236.

18. Silva JL, De Moura Gallo CV, Costa DC, Rangel LP. Prionlike aggregation of mutant p53 in cancer. Trends Biochem Sci. 2014; 39:260-267.

19. Gurpinar E, Vousden KH. Hitting cancers' weak spots: vulnerabilities imposed by p53 mutation. Trends Cell Biol. 2015; 25:486-495.

20. Muller PA, Vousden KH. Mutant p53 in cancer: new functions and therapeutic opportunities. Cancer Cell. 2014; 25:304-317.

21. Cooks T, Pateras IS, Tarcic O, Solomon H, Schetter AJ, Wilder S, Lozano G, Pikarsky E, Forshew T, Rosenfeld N, Harpaz N, Itzkowitz S, Harris CC, et al. Mutant p53 prolongs NF-kappaB activation and promotes chronic inflammation and inflammation-associated colorectal cancer. Cancer Cell. 2013; 23:634-646.

22. Shi L, Jackstadt R, Siemens H, Li H, Kirchner T, Hermeking H. p53-induced miR-15a/16-1 and AP4 form a doublenegative feedback loop to regulate epithelial-mesenchymal transition and metastasis in colorectal cancer. Cancer Res. 2014; 74:532-542. 